This item was submitted to Loughborough's Research Repository by the author.

Items in Figshare are protected by copyright, with all rights reserved, unless otherwise indicated.

\title{
Factors affecting accuracy in the quality control checking of fresh produce labels: A situational and laboratory-based exploration
}

PLEASE CITE THE PUBLISHED VERSION

https://doi.org/10.1002/hfm.20806

\section{PUBLISHER}

(c) Wiley

\section{VERSION}

AM (Accepted Manuscript)

\section{PUBLISHER STATEMENT}

This is the peer reviewed version of the following article: SMITH-SPARK, J.H. ... et al, 2019. Factors affecting accuracy in the quality control checking of fresh produce labels: A situational and laboratory-based exploration. Human Factors and Ergonomics in Manufacturing and Service Industries, 29(6), pp. 447-458, which has been published in final form at https://doi.org/10.1002/hfm.20806. This article may be used for non-commercial purposes in accordance with Wiley Terms and Conditions for Use of Self-Archived Versions.

\section{LICENCE}

CC BY-NC-ND 4.0

\section{REPOSITORY RECORD}

Smith-Spark, James H., Hillary B. Katz, Thom Wilcockson, and Alexander P. Marchant. 2019. "Factors Affecting Accuracy in the Quality Control Checking of Fresh Produce Labels: A Situational and Laboratorybased Exploration". figshare. https://hdl.handle.net/2134/38062. 
Running Head: Factors affecting label-checking accuracy

Factors affecting accuracy in the quality control checking of fresh produce labels: A situational and laboratory-based exploration

\author{
James H. Smith-Spark ${ }^{1}$, Hillary B. Katz ${ }^{1}$, Thomas D. W. Wilcockson ${ }^{2}$, and Alexander P. \\ Marchant $^{1}$ \\ ${ }^{1}$ London South Bank University, London, United Kingdom \\ ${ }^{2}$ Loughborough University, Loughborough, United Kingdom
}

\begin{abstract}
Author Contact
James H. Smith-Spark, Division of Psychology, School of Applied Sciences, London South Bank University, 103 Borough Road, London, SE1 0AA, United Kingdom

Emailsmithspj@1sbu.ac.uk
\end{abstract}

Telephone +44 (0)20 78155884

Fax +44 (0)20 78158099 


\begin{abstract}
Manufacturing industries often rely on quality control staff to ensure mistakes are detected before products are shipped to customers. Undetected errors can result in large financial and environmental costs to packaging companies and supermarkets but the contributors to such error are underexplored. The research reported in this paper investigated human error in the quality control checking of information displayed on the labels which accompany packaged fresh produce. Initial work sought to understand the demands of label-checking in the packhouse environment, through interviews with key quality control staff, in situ observations, and the study of historical error data held by a fresh produce packaging company. This work highlighted the dynamic and cognitively challenging environment in which label-checking occurred, while the historical error data indicated both the scale of the packhouse's work and the infrequency of error occurring. In a separate strand of laboratory-based research, experienced and novice labelcheckers were presented with a simulated label-checking task and a battery of computerised and pen-and-paper tests. These tasks were administered to determine whether cognitive abilities could predict label-checking accuracy in a controlled laboratory environment. Stronger abilities in two cognitive processes (information processing speed and inhibition) predicted greater overall accuracy and higher detection of labelling errors. In identifying potential contributors to human error in the quality control checking of product labels both in situ and in the laboratory, the results are relevant to manufacturing, wherever information is printed on labels, especially when labelling processes depend upon human data entry and human quality control checking. Keywords: Cognition; Human Error; Individual Differences; Quality control; Visual Inspection
\end{abstract}




\section{INTRODUCTION}

The labels printed on packaging containing fresh fruit and vegetables are checked by human operatives before consignments are transported from packaging facilities via distribution depots to supermarket retailers. This quality control procedure is set in place to ensure that the information printed on the label corresponds exactly with the contents of the packaging in terms of product details and price. Despite several independent checks by quality control staff being conducted as a matter of routine, very occasionally labelling errors fail to be detected. In such cases, the errors are usually discovered too late to remedy, being discovered only after the production run has been distributed to retail outlets. Such errors have very significant consequences for packaging companies and supermarkets alike, in terms of reputational and financial costs and environmental impact, since the affected produce will need to be recalled for repackaging, replacement or, sometimes, disposal. As well as supplying the consumer with the correct information upon which to base a decision as to whether to buy the product, there can also be potential legal and health implications if the information presented is incorrect. To reduce the frequency of labelling errors that pass quality controls unnoticed, it is necessary to understand the processes involved in checking product labels, both with respect to the quality control system in operation and the cognitive demands that label-checking entails. In broad terms, the quality control checking of product labels can be regarded as a type of visual inspection task in which products are checked for correctness by eye. Visual inspection tasks are well studied in the ergonomics literature (e.g., Liu \& Yu, 2018), across a range of different industrial, contextual, and organizational settings. These include aircraft inspection (Drury, 1993), airport x-ray security inspection (Ghylin, Drury \& Schwaninger, 2006), telecommunications (Jameeson, 1966), inspection of small steel cylinders (Kleiner \& Drury, 
1993), the use of video magnifiers for inspecting electronic and mechanical components of consumer products (Lee, Man, \& Chan, 2019), pharmaceutical and biological drug products (Melchore, 2011), contact lens inspection (Rao, Bowling, Khasawneh, Gramopadhye \& Melloy, 2006), circuit patterns, sheet steel or glass inspection, circuit board comparison (Wang \& Drury, 1989), and simulated solder joint arrays (Wang, Lin \& Drury, 1997). However, despite the costly nature of human error in this domain, only a small amount of direct research has been carried out on product label-checking (Smith-Spark, Katz, Marchant \& Wilcockson, 2015, 2016; SmithSpark, Katz, Wilcockson \& Marchant, 2018). The research reported in this paper focused on one fresh produce packaging company which served a range of UK supermarkets. It had two broad aims: firstly, to identify situational contributors to error in the quality control checking of labels in the packhouse environment and, secondly, to explore cognitive factors that might predict label-checking accuracy under controlled laboratory conditions.

The paper begins by describing the processes involved in the packaging of fresh produce and the cognitive demands placed on the operatives involved in the quality control checking of product labels. Potential contributors to human error in quality control checking were investigated in the packhouse environment through interviews with key operatives, in situ observations, and the study of historical error data held by the packaging company. To determine whether a range of cognitive abilities predicted label-checking performance in the laboratory, the results of a simulated label-checking task are then reported.

\subsection{The process of packaging and labelling fresh produce}

At the packaging facility involved in the current research, fresh produce is prepared in the packhouse for transportation from the packaging facility to distribution depots and, from these depots, to supermarkets. Over 170 fresh produce Stock Keeping Units (SKUs) per day are 
processed by the packaging facility. The packaging facility's work is made even more complex by variations in the size, variety, and grower of the produce when the fresh fruit and vegetable orders are packaged. Study of the historical records held by the packaging facility indicated the high volume of orders that it processed, There were, for example, 1316 label runs for stone fruit alone in the month of March 2014.

On a weekly basis, the commercial team of a supermarket chain contacts the packaging facility's commercial office with a list of updates to their fresh produce requirements (such as size changes, promotions, etcetera) relating to the product in question. This information is then entered into a product specification spreadsheet and checked by two people against the original order as specified in the email or telephone communication from the supermarket.

After this checking has occurred, the product specification sheet is communicated to packaging line leaders and label print room staff in the packaging facility (or "packhouse"). The information that it provides is used by packhouse operatives to make up the orders correctly before they are shipped to the supermarket customer. The product specification sheet is updated as and when necessary during its weeklong lifespan. At the end of the week, it is replaced by an entirely new version. An example of a simplified product specification sheet is shown in Figure 1.

\section{FIGURE 1 ABOUT HERE}

Packaging line leaders are in charge of teams of operatives responsible for processing orders on a particular packaging line. For each order processed by the line, the packaging line leader generates a label check sheet, checking it against the quantity or size of the fresh produce required, the list of growers approved by the supermarket, and the varieties of fresh produce approved by the supermarket in question. In line with the packaging line leader's requirements, 
the required quantities or weights of loose fresh produce currently held in store in the facility's warehouse are transported to the packaging line. Once there, they are packaged to meet the supermarket's order as set out on the product specification sheet (e.g., six apples per pack or $400 \mathrm{~g}$ of grapes per punnet). Depending on its type, fresh produce is packaged in different ways. For example, fruit and vegetables may be packed in punnets with a label printed on a film seal, sealed in polythene packets or netted.

As a part of the packaging process, labels are printed to accompany each unit making up the order in question. With the exception of fresh produce packaged in film seals (which require their own dedicated printing machines as an integral component of the packaging line), product labels need to be printed to accompany the packaged units of produce. A run of printed labels is, therefore, ordered by the packaging line leaders to fulfil their current packaging job. Staff in the label room staff receive these orders and are responsible for printing the labels.

Each fresh produce label has between three and 11 fields of information. It provides details on, for example, the type of produce, quantity or weight, best before date, grower name, and country of origin. A further ribbon or sticker, known as a "flash label", may accompany the label in the event that there is a current promotion being offered on the product (e.g., "Buy 1, get 1 free" or " 2 for $£ 2.50 ”)$. An example of such a label is shown in Figure 2. The line leader then collects the printed labels from the label room.

\section{FIGURE 2 ABOUT HERE}

In order to ensure that the information presented on the label corresponds with both the product specification sheet and the contents of the packaged item, checks are made at the beginning and end of the packaging run. In checking a label, a quality control operative must move visually between two sources of information, verifying that the information in each field of 
the label matches exactly its corresponding entry on the product specification sheet. Observation of label checks performed in the packhouse indicated that it took around 20 s to complete a label check. The outcomes of the checks are recorded on a checklist which the quality control checkers must sign to indicate that the check has taken place- As part of this signing-off process, the checker must attach an example label to the completed checklist and highlight any label errors which have been detected. In the event of an error being discovered, action appropriate to the stage at which it is discovered is taken to correct it (i.e., reprinting labels or repackaging incorrectly labelled products). Three or four independent quality control checks are, therefore, conducted over the course of packaging the fresh produce.

Despite this rigorous quality control process, label printing errors still occasionally escape detection. The historical error records held by the packaging company showed that for the month of March 2014, fewer than 1\% of processed orders contained errors and, of this $1 \%$, less than $5 \%$ were undetected during quality control checking (i.e., approximately five in every 10,000 label runs contain errors that go undetected). There is, thus, a disproportionately large effect of a very small number of undetected errors on the packaging company's reputation and finances. Indeed, while no figures appear in the public domain for verification, failures to detect labelling errors have been estimated by a senior industry source to cost the UK supermarket industry some $£ 8-10 \mathrm{~m}$ per annum in repackaging, recalls, and fines (S. Hinks, Product Technical Manager: Fruit and Floral, Sainsbury's Supermarkets Ltd, personal communication).

A flowchart depicting the label-checking process is presented in Figure 3, giving task descriptions, identifying the task performer, and the person in charge of a particular stage. FIGURE 3 ABOUT HERE. 


\section{UNDERSTANDING THE DEMANDS OF LABEL-CHECKING IN THE PACKHOUSE ENVIRONMENT}

To obtain a greater understanding of the likely contributors to label-checking error in the packhouse, individual structured interviews were conducted with 17 quality control staff across three of the packaging company's facilities. The questions asked the interviewees to consider their levels of motivation, sense of responsibility, physical wellbeing, and mental alertness in relation to the incidence of errors. During the interviews, the participants were asked to identify the factors which they felt contributed to label-checking error. These factors are summarized in Table 1 in which the percentage of interviewees identifying a given factor as a contributor to error is indicated. In the following text, these factors are linked, where relevant, to cognitive psychological research and theory.

\section{TABLE 1 ABOUT HERE}

As well as items relating to the impact of state of personal wellbeing, time pressure, and workload (with either having too much or too little to do perceived as resulting in more error; suggesting an optimal level of arousal; cf. the Yerkes-Dodson Law; Yerkes \& Dodson, 1908), several cognitive factors contributing to error could be identified in the comments provided by the quality control operatives. These will now be considered in more depth.

Cognitive load (e.g., Sweller, 1988), reflected in the number of bits of information which needed to be checked on the label, was identified by $71 \%$ of the interviewees as leading to error. Given that information has to be held in temporary memory while it is checked for equivalence across two printed sources, label-checking is also likely to require short-term memory resources (e.g., Cowan, 2008). Digit span has previously been identified as a positive predictor of labelchecking accuracy under laboratory conditions in university students (Katz et al., 2015) and, 
more generally, it has been found to predict visual inspection abilities on other tasks (Gallwey, 1982; Wang \& Drury, 1989; but see Wang et al., 1997). These findings would seem to corroborate the argument that label-checking, at the very least, draws on similar cognitive processes to visual inspection. However, compared with visual inspection in other industrial domains (e.g., Drury, 1993; Ghylin et al., 2006; Jameeson, 1966; Melchore, 2011; Rao et al., 2006; Wang \& Drury, 1989; Wang et al., 1997), label-checking would seem to place greater demands on short-term memory resources (e.g., Cowan, 2008). This is due to information having to be compared across two sources, namely the label and the product specification sheet which provides details of the retailer's order. In many other visual inspection tasks involving manufactured goods, the checking process would be more likely to require the comparison of an exemplar against a "standard" representation of the desired appearance of the product held in long-term memory, rather than a direct comparison of one physical object or image with another as is the case with label-checking.

High noise levels in the packhouse were highlighted by $65 \%$ of the interviewees as a contributor to label-checking error. Taylor, Melloy, Dharwada, Gramopadhye, and Toler (2004) have highlighted the varying effects of noise from a single source on visual inspection depending on task complexity. Although not tested directly in their study, Taylor et al. argued that noise emanating from multiple sources is likely to have deleterious effects on visual inspection when performing complex visual search tasks. The reports of the interviewees would seem to support this argument.

Over half the interviewees (53\%) suggested that the use of coloured fields to distinguish entries on the product specification sheet would make it easier to read off the required information and, as a result, reduce error compared with the current use of black and white 
fields. Visual search processes (e.g., Eckstein, 2011) are the first step in identifying a target item or a defect in a visual inspection task (e.g., Melloy, Harris \& Gramopadhye, 2000; Taylor et al., 2004). They are likely to be drawn upon during label-checking when the individual actively scans the the product specification sheet and the product label for pertinent information amongst an array of other, currently non-relevant, information (e.g., finding the entry for the product's best before date amongst information on the product name, grower name, product weight, and barcode). However, despite visual search seeming an obvious candidate as a cognitive predictor of label-checking performance, previous research has found that it did not significantly predict overall label-checking accuracy (Katz, Smith-Spark, Wilcockson \& Marchant, 2015).

Fifty-nine per cent of the interviewees identified interruptions by colleagues working on the packaging line as a contributor to label-checking error. A review of the effects of interruptions in the workplace is provided by Trafton and Monk (2007). In the event of an interruption, individuals need to remember where they left off from a task so that they can then resume it at the correct point and avoid missing or mistaking important intermediate steps in the task procedure. Prospective memory, which is memory for delayed intentions (Winograd, 1988) or "remembering to remember" (Mäntylä, 1994), is also highly likely to be involved. Task interruptions would load on prospective memory resources, with an intention having to be formed to pick up the interrupted task at the point at which it was left off and the intention being acted upon when task performance can be resumed (e.g., Dodhia \& Dismukes, 2009; Wilson, Farrell, Visser \& Loft, 2018).

Further to the interviews conducted with quality control staff, open-ended discussions were also held with the packaging facility's management. These emphasized the serious repercussions (identified in terms of business reputation, financial cost, and environmental 
impact) arising from label errors going undetected, despite the infrequency of their occurrence. Moreover, these discussions highlighted particular concerns over errors arising from the checking of the flash labels accompanying the packaged product to highlight a current promotional offer on the product in question (see Figure 2 for an example). The management of the packaging facility attributed approximately $25 \%$ to $50 \%$ of quality control errors to checks carried out on the flash label. This is a disproportionate amount, given that they are one bit of information to check amongst three to 11 fields of information presented on the label. There are serious consequences in terms of customer confusion, supermarket reputation, and cost if the mislabeled product reaches the retailer undetected, only to be discovered when the erroneous price on the label fails to match the supermarket's records when being rung through the tills. As promotional offers on products are seasonal, the flash label does not appear on all product lines that are currently being packaged. Moreover, flash labels have both a supermarket-defined date at which they should begin to accompany the product packaging and a supermarket-defined date on which the promotional offer ends, at which time the flash label should no longer appear on the packaging. This variability in the presence of flash labels and the nature of the current offer that they highlight presents a particular challenge to label-checkers. Failure to detect flash label errors is likely often to represent a premature exit from label-checking before the flash label is checked. Label-checking errors affecting flash labels may thus be explained in terms of postcompletion errors (Byrne \& Bovair, 1997). This type of error occurs when the primary goal of the task is achieved (i.e., checking the product label itself) but the final step (or steps) in the procedure are omitted (i.e., failing to check the accompanying flash label).

These then are the situational factors identified by key operatives and through observations as contributing to human error in the quality control checking of fresh produce 
labels. The second strand of the research sought to explore label-checking performance in the laboratory setting and to determine whether individual differences in a range of cognitive abilities would predict label-checking accuracy.

\section{LABEL-CHECKING PERFORMANCE UNDER LABORATORY CONDITIONS}

To date, only the contribution of individual differences in basic cognitive processes to label-checking performance has been studied under laboratory conditions (Katz et al., 2015). The cognitive processes investigated so far have been short-term memory, perceptual comparison accuracy, and attention. Of these, verbal short-term memory was found to be the only significant predictor of label-checking accuracy. The laboratory study reported in the current paper, therefore, extended this work to explore a range of further cognitive abilities. The choice of abilities tested will now be considered.

The ability to work successfully in the dynamic packhouse environment would suggest the need for higher-order cognition, or executive functions (e.g., Diamond, 2013; Miyake et al., 2000), to be exercised in keeping quality control checkers "on task" in the face of frequent distractions and interruptions. More specifically, better abilities to work in the face of distraction, to switch efficiently between tasks, and smoothly to resume previously initiated cognitive operations as required after interruptions from colleagues would all prove advantageous to quality control operatives. In the current study, measures were thus taken of the executive functions of updating, inhibition, and set shifting. This choice of measures also allowed theoretical coherence to the research, since these three make up a tripartite model of executive functioning that is well-established in the cognitive psychological literature (Miyake \& Friedman, 2012; Miyake et al., 2000) and in which individual differences are well-documented (Friedman \& Miyake, 2017). Updating refers to the ability to update information in memory in 
the light of new information (e.g., Miyake et al., 2000) and has been argued to engage the same cognitive processes as working memory (Chein, Moore \& Conway, 2011). Set shifting (e.g., Monsell, 2003) describes the ability to move fluently and efficiently between different cognitive operations or sets. Inhibition relates to the ability to inhibit habitual or pre-potent responses in favor of more task-appropriate behaviour (e.g., Miyake et al., 2000).

While perceptual comparison speed had not previously been found to be a significant predictor of label-checking accuracy (Katz et al., 2015), this is just one type of basic information processing ability. The rate at which individuals could convert one form of information into another was, therefore, also assessed in the current study using the Digit-Symbol subtest from the Wechsler Adult Intelligence Scale - Third Edition (WAIS-III; Wechsler, 1998).

Level of alertness or fatigue had been identified by interviewees as a contributory factor to error and was directly open to testing using a well-established self-report measure of participant alertness. The Karolinska Sleepiness Scale (KSS; Åkerstedt \& Gillberg, 1990) was, therefore, administered to explore this potential contributor to error under laboratory conditions.

Past visual inspection work has indicated that inexperienced students tend to perform at a similar level to that of individuals who have had experience with the task through their job role (Wang et al., 1997). Consistent with this finding, Smith-Spark et al. (2018) also found that the number of years of experience in label-checking was not related to either the accuracy of performance or how strategic checkers were in their approach to the task. The contribution of prior experience to performance was investigated further in the current study by determining whether group differences existed between professional label checkers and university students naïve to the process of label-checking in label-checking accuracy. 
Following this group comparison, the data from both participant groups were combined to allow a sufficient $N$ to permit the use of multiple regression analyses to explore whether labelchecking accuracy could be predicted reliably on the basis of cognitive abilities (updating, inhibition, and set shifting) and perceived alertness (the KSS; Åkerstedt \& Gillberg, 1990).

\section{1 Method}

\subsubsection{Participants}

A total of 32 individuals took part (mean age $=26$ years, $S D=7$ ). Of these, 16 were experienced label checkers (mean age $=30$ years, $S D=6 ; 12$ females, 4 males; mean years of experience $=5$ years, $S D=4$ ). The experienced label checkers were employees at a large fresh produce packaging facility and their job description included responsibility for the quality control checking of product labels. They undertook the study as part of their regular working week, being paid their regular hourly rate, and were reimbursed travel expenses by their employer for attending the laboratory for testing. The experienced label checkers did not perform a shift on the same day as the laboratory testing took place. A further 16 participants formed a novice group of university students who reported themselves to be naïve to the process of quality control checking fresh produce labels (mean age $=23$ years, $S D=7 ; 13$ females, 3 males). The university students received either course credit or a modest honorarium in appreciation of their time.

\subsubsection{Materials and tasks}

\subsubsection{Measures of sleepiness and cognition}

The KSS (Åkerstedt \& Gillberg, 1990) measures subjective levels of sleepiness. Respondents use a nine-point scale $(1=$ extremely alert, $3=$ alert, $5=$ neither alert nor sleepy, 7 
$=$ sleepy - but no difficulty remaining awake, and $9=$ extremely sleepy - fighting sleep) to indicate the level best reflecting their sleepiness in the last 10 minutes.

The Go/No Go task (Luria, 1966) was employed as a measure of inhibition. Two $256 \mathrm{x}$ 256-pixel greyscale line drawings, matched for visual complexity, were taken from a wellestablished picture set (Snodgrass \& Vanderwart, 1980). One of these line drawings was used as the "habituated" stimulus, requiring participants to press a key whenever it was presented. The second line drawing was employed as the stimulus requiring a less frequently occurring (and, thus, less anticipated) response. In contrast to the key press required by the habituated stimulus, the participants were instructed to refrain from making any key press to this non-habituated stimulus. In order to build up anticipation of an expected (or pre-potent) response, the initial 40 trials of the task consisted entirely of stimuli which required the motor response to be made. After this habituation phase, the experiment shifted to an inhibition phase without the participant being made aware of this change. The 160 trials of the inhibition phase consisted of a combination of habituated and non-habituated trials, in the ratio of four to one. The nonhabituated trials were presented in a pseudo-randomized fashion, appearing every three to six trials. The same ratio of trial types has been used in other published research (e.g., Smith-Spark, Henry, Messer, Edvardsdottir \& Zięcik, 2016; Smith-Spark, Moss \& Dyer, 2016). The number of occasions on which the participant managed successfully to withhold a motor response to the non-habituated stimulus was recorded and expressed as a percent accuracy value. Higher accuracy scores indicated better inhibitory abilities.

The Plus-minus task (Jersild, 1927) was used to measure set shifting. Three printed lists were presented, each consisting of 30 two-digit numbers (drawn from the numbers 10-99, prerandomized without replacement). Each list was presented on a separate printed page. On the 
initial Plus-3 trial, the participants were instructed to add three to each two-digit number that was presented and to write their answers next to the number in question. There then followed the Minus-3 trial, in which the participants were instructed to subtract three from each two-digit number. Finally, in the Plus-Minus condition, the participants were required to alternate between the two mathematical operations. The participants were instructed to complete each list of the Plus-minus task as quickly and accurately as possible. List completion times were recorded using a stopwatch. A mean of the times to complete the Plus-3 and Minus-3 conditions was calculated. This mean was then subtracted from the time to complete the Plus-Minus condition to give the cost of shifting between the two mathematical operations rather than remaining "in set".

The automated version of the Operation Span (e.g., Unsworth, Heitz, Schrock \& Engle, 2005) was used to measure updating. The participants were asked to indicate with a Yes/No keypress whether a given number displayed on a computer screen was the correct answer to a simple arithmetic problem. After giving their answer, an alphabetical letter was presented and the participants were asked to retain the letter in memory for later recall. The number of arithmetic problems presented before serial recall of the letters was required varied between three and seven, with there being three trials at each list length. Absolute span was recorded for later analysis. This measure corresponded to the number of trials on which the participants correctly recalled all letters in the correct serial order.

The Digit-Symbol subtest was taken from the WAIS-III (Wechsler, 1998). The participants were given a key containing nine digit-symbol pairs. A worksheet containing a number of rows of symbols, each with empty boxes underneath, was presented below this key. The participants were asked to use the key to write the appropriate digit (e.g., "2") in the empty box which was associated with the symbol displayed above it (e.g., "\%”). The standard 
administration of the task requires participants to complete as many of the digit-symbol pairs as they can in two minutes; however, in the current study, the participants were timed to complete the entire 120 stimulus pairs instead.

\subsubsection{Simulated label-checking task}

Experimenter Builder Version 1.4.128 B (SR Research Ltd., Ontario, Canada) was used to control the stimulus events during the label-checking task. One hundred fresh produce labels were provided by the packaging company. Each label contained the following seven fields of information: product name, grower, variety, quantity, best before date, barcode, and a flash label indicating current promotional activity. The same seven fields of information were also presented in a corresponding product specification sheet containing entries for five products, one to a row. The label stimuli were presented on the lower half of a computer monitor and their sizes varied depending on label-type. The specification sheets were displayed on the top half of a computer monitor and were the same size across trials.

A gaze-contingent paradigm was used to simulate the process of moving between the label and the product specification sheet. When the participants' eyes were fixated on the tophalf of the screen, only the label was viewable, but when the participants' gaze fixated on the bottom-half of the screen, the product specification sheet alone was visible. The dominant eye of each participant was determined using the Miles test (Roth, Lora \& Heilman, 2002).

The participants were informed that they would be presented with a series of trials in which product labels and corresponding product specification sheets would be displayed on the computer monitor. On each trial, they were asked to indicate with a Yes/No key-press whether or not the information shown on the label matched the corresponding information for that product presented on the product specification sheet. The participants were told that they could take as 
long as they liked to arrive at their decisions. Two 50-trial blocks were presented to participants. Each block contained 40 congruent trials and 10 non-congruent trials (where one field of information differed between the label and the product specification sheet).

\subsubsection{Design}

Two-way mixed-measures analyses of variance (ANOVAs) were performed to determine whether the label-checking performance of the experienced and novice label-checkers differed. The between-subjects factor was participant group (levels: experienced, novice) and the withinsubjects factor was block of trials (Block 1, Block 2). The dependent variables were overall percentage accuracy of response (regardless of trial type; i.e., correctly stating either that a label matched the product specification sheet or that it did not) and the percentage of label errors detected (i.e., correctly stating that there was a mismatch between the information printed on the label and that displayed on the product specification sheet).

To determine whether group differences in KSS scores and the cognitive measures might explain any differences in label-checking performance, a multivariate analysis of covariance (MANCOVA) was run, with age entered as the covariate due to the group difference in age that was reported in Section 3.1.

After this, predictors of label-checking performance (i.e., Go/No Go accuracy, Plusminus shift cost, Operation Span score, Digit-Symbol raw score, and KSS score at Time 1) were entered into two stepwise multiple regression analyses. Overall accuracy and the percentage of label errors which went undetected were entered as the respective output variables.

\subsubsection{Procedure}

Full ethical approval was granted by the relevant ethics committee at the first author's host institution. The participants gave informed consent to take part. The tasks were presented in 
the following order: the first administration of the KSS, the label-checking task instructions and visual search task, the first 50-trial block of the label-checking task, the operation span task, the go/no-go task, the plus-minus task, the Digit-Symbol task, the second administration of the KSS, and the second 50-trial block of the label-checking task. Testing was performed on an individual basis, taking approximately two hours per participant. Each block of label-checking trials took approximately 30 minutes for the participants to complete, with there being an interval of around 60 minutes between blocks, during which the cognitive tests and the KSS were presented. The participants were debriefed at the end of the testing session.

\subsection{Results}

3.2.1 Comparing the label-checking performance of experienced label-checkers and novices

\subsubsection{Overall accuracy}

A two-way mixed-measures ANOVA was conducted on overall label-checking accuracy. No statistically significant difference was found between the experienced label-checkers $($ mean $=92.25 \%, S E M=1.20)$ and the novices $($ mean $=91.50 \%, S E M=1.20), F(1,30)<1, M S E$ $=45.93, p=.661$. Accuracy in Block $1($ mean $=90.81 \%, S E M=1.14)$ was slightly lower than in Block $2($ mean $=92.94 \%, S E M=0.88)$ but the difference was not significant statistically, $F(1$, $30)=3.51, M S E=20.58, p=.071$. There was no significant interaction between participant group and block of trials, $F(1,30)<1, M S E=20.58, p=.743$.

\subsubsection{Detection of label errors}

The experienced label-checkers $($ mean $=91.88 \%, S E M=2.39)$ detected a greater percentage of errors than the novice group (mean $=80.00 \%, S E M=2.39)$. The effect of participant group was very significant statistically, $F(1,30)=12.34, M S E=182.92, p=.001, \eta_{\mathrm{p}}^{2}$ $=.291$. Participants detected a greater percentage of label errors in Block 1 (mean $=90.00 \%$, 
$S E M=1.91)$ than in Block $2($ mean $=81.88 \%, S E M=2.30)$. Block was also found to have a very significant effect on error detection, $F(1,30)=10.26, M S E=102.92, p=.003, \eta_{\mathrm{p}}{ }^{2}=.255$. There was also a significant participant group $\mathrm{x}$ block of trials interaction, $F(1,30)=10.26, M S E=$ 102.92, $p=.003, \eta_{\mathrm{p}}{ }^{2}=.255$. The interaction is plotted in Figure 4. Post hoc t-tests indicated that there was no significant difference between the experienced label-checkers and the novices in Block $1, t<1, p=.333$, but that the novices missed significantly more label errors in the Block 2 than the experienced group, $t=4.34, p<.001$, Cohen's $d=4.35$. Moreover, while there was no significant difference between blocks for the experienced label-checkers, $t<1, p=1.00$, the novices' performance in Block 1 was significantly better than in Block 2, $t(15)=4.10, p=.001$, Cohen's $d=1.02$.

\section{FIGURE 4 ABOUT HERE}

\subsubsection{Comparing group scores on the predictor variables}

The change in KSS scores between the first and second blocks was analyzed. Over time, the novice group reported a relatively greater change towards sleepiness (mean change $=0.88$, $S D=1.15)$ than the experienced label-checkers (mean change $=0.13, S D=1.15)$. However, this difference was found to be statistically non-significant, $t(30)=1.85, p=.074$.

Table 2 shows the group mean scores for all the measures entered into the regression models. The MANCOVA indicated no significant multivariate effect of group on the measures, Wilks' $\Lambda=.673, F(5,25)=2.43, p=.063$.

\section{TABLE 2 ABOUT HERE}

\subsubsection{Predictors of label-checking performance}

The remaining analyses were focused on exploring predictors of label-checking performance in Block 1, when there was no difference between the two groups in the levels of 
fatigue nor in accuracy of performance (experienced label-checkers: mean accuracy $=91 \%, S D=$ 6; novices: mean accuracy $=90 \%, S D=6), t(30)<1, p=.625$. There was also no significant difference in the percentage of Block 1 trials on which a label "error" failed to be detected between the novices (mean $=12, S D=10)$ and the experienced label-checkers (mean $=8, S D=$ $11), t(30)<1, p=.333$.

A stepwise regression indicated that the model significantly predicted overall labelchecking accuracy, $R=.386$, adjusted- $R^{2}=.119, F(1,29)=5.07, p=.032$. Only Go/No Go accuracy was entered into the final model as a significant predictor, $t=2.25, p=.032$. Better inhibitory abilities were associated with higher label-checking accuracy. Table 3 shows the partial correlations, $t$-scores, and $p$-values for the predictor variables entered into the stepwise regression.

\section{TABLE 3 ABOUT HERE}

When the accuracy of detecting the presence of label errors was considered, a stepwise regression again indicated that the model significantly predicted performance, $R=.464$, adjusted- $R^{2}=.188, F(1,29)=7.94, p=.009$. Of the individual predictors, only Digit-Symbol raw score was entered into the final model as a significant predictor of poorer performance, $t=$ $2.92, p=.007$. Slower information processing speed was associated with a higher percentage of failures to detect the presence of a label error. The test statistics for all of the predictor variables are shown in Table 4.

TABLE 4 ABOUT HERE

\subsection{Discussion}

A simulated label-checking task was presented to experienced and novice label-checkers. The stimuli consisted of labels and a corresponding specification sheet, against which the 
accuracy of information had to be checked. The task closely resembled the parameters of work in an actual packaging facility, both in terms of the physical features of the materials being processed and the behavioural requirements of the task as undertaken by quality control staff there. On average, label checks took approximately 20 s to complete, matching the comfortable rate of label-checking observed on the packhouse floor and reported in interviews with key operatives. The simulated task was also similar to the real-world situation in that label 'errors' were programmed to occur unpredictably as far as the participant was concerned.

Overall label-checking accuracy was found to be high and there was no main effect of level of experience on performance. However, level of experience was found to interact with experimental block. While no group difference was found in Block 1, the experienced labelcheckers maintained a higher level of accuracy in Block 2 in relation to the novice group whose performance dropped considerably. The decline in the performance of the novice group suggests a difference in attention, motivation or fatigue, most likely arising from having less practice with the task demands. While statistically non-significant, the difference between the groups in KSS scores (Åkerstedt \& Gillberg, 1990) might provide tentative support for this argument. The experienced label-checkers undertook the task as part of their regular working week and, thus, were highly likely to have felt a professional pride in their performance and a responsibility to carry out the label-checking at a consistently high level throughout testing. Conversely, the novice participants, carrying out the task for course credit or payment of a small honorarium, may have lost motivation over the course of the study, due to its relatively long duration and the lack of variation in what was required of them, or may have been more affected by fatigue due to the unfamiliar nature of the task. These factors may have led to the reduction in label error detection accuracy in Block 2. Since no group differences were found in Block 1, the data were 
collapsed across experienced and novice label-checkers to identify cognitive predictors of labelchecking accuracy when both groups were fresh to the task and no group differences were evident in accuracy of performance.

Scores on two cognitive measures were found to predict accuracy on the simulated labelchecking task and these predicted performance on different dependent measures. These were Go/No Go accuracy (reflecting inhibitory abilities; e.g., Miyake et al., 2000) and WAIS-III Digit-Symbol score (reflecting information processing speed; Wechsler, 1998). On the other hand, no significant predictive relationships were found between the indices of label-checking performance and Operation Span score (which provided a measure of updating), Plus-Minus switch cost (which gave an index of set shifting) or KSS score (which indicated self-perceived level of alertness).

A stronger ability to inhibit pre-potent responses was positively associated with overall accuracy. More accurate performance on the Go/No Go task was related to greater labelchecking accuracy. Given that only $20 \%$ of trials contained a label error, the more frequent (and, thus, the more expected response) would be "Yes, the two sources match". Label checking, too, requires vigilance on the part of quality control operatives in that they have to respond differently to relatively infrequently occurring events (i.e., label errors) against a backdrop of more commonly occurring events (i.e., matches between the label and the entry on the product specification sheet). The positive association between Go/No Go accuracy and the overall accuracy of label-checking performance is thus likely to reflect the need to inhibit the pre-potent assumption that all fields are correct (as they were on $80 \%$ of the trials in the present study) and, instead, identify the presence of an error and respond accordingly. This conclusion is likely to be 
relevant to other domains of industry or commerce where mistakes which need to be detected occur rarely over long series of repetitive presentations.

At a finer-grained level of analysis, focusing on label error detection only, participants who exhibited a faster speed of information processing missed fewer label errors than participants who took longer to complete the Digit-Symbol task. The ability to process information quickly would thus appear to be beneficial to label-checking performance. This may reflect a reduction in errors relating to short-term memory (identified previously as a positive predictor of label-checking accuracy by Katz et al., 2015), given that there would be less time over which relevant label details could decay during a visual search for a particular field of information.

Future research should explore the role of executive functioning abilities in labelchecking performance in the presence of environmental distractors (such as background noise or staged interruptions). Given the controlled and predictable laboratory environment in which the simulated label-checking task was conducted, one might expect an increase in the predictive power of these variables under the noisy and dynamic real-life conditions highlighted in the interviews with key operatives. In addition, the use of physical printed labels and product specification sheets would add to the ecological validity of the research (although the use of an eye-tracker might be curtailed by the use of physical stimuli rather than virtual analogues presented on the computer screen). Exploring performance using physical stimuli rather than virtual analogues would seem to present the next step in the research of label-checking. Finally, the number of fields of information requiring checking has been purposefully restricted to seven for two reasons. Firstly, previous work has shown that a larger amount of information to inspect is likely to result in poorer performance (Gallwey \& Drury, 1986) so inter-trial variation in the 
number of fields to be checked has been avoided in the label-checking studies to date (Katz et al., 2015; Smith-Spark et al., 2015, 2016, 2018). Secondly, poorer visual inspection performance has been found when errors are present in multiple fields rather than in single fields (see Gallwey \& Drury, 1986, for a review). In the packhouse, the number of fields of information presented on labels varies between three and 11 depending on the product and errors may occur in two or more fields of information on any given label. Future research should, therefore, seek to explore the effect on performance of label 'errors' occurring in multiple fields of the same label and with varying numbers of fields requiring checking.

The same limitations identified by Smith-Spark et al. (2018) apply to the laboratory work reported in this paper. The simulated label-checking task differed from the task as it is performed in the packhouse in several ways. Presentation of stimuli was computer-based rather than requiring interaction with physical labels and product specification sheets, with a considerably higher frequency of errors than that occurring in the packhouse in order to yield sufficient data points for work conducted in a laboratory setting. This compromise over the frequency of errors was needed to manage the time and resource constraints under which the research team and the packhouse operated and also to retain the goodwill of all the participants. Indeed, the decline in performance of the novice participants and the drop in their KSS scores over a two-hour period of testing would seem to argue strongly against the use of a more realistic ratio of labels containing errors (and the extended duration of testing that this would require). Finally, labelchecking trials followed immediately after each other. In the packhouse, label-checking would be one task performed around a range of other professional duties, often with distractions occurring. If the end of different production runs coincided, it might be the case that several label checks would need to be carried out by the same quality control checker in quick succession but, even 
when the packhouse was at its busiest, not to the quantity presented in the laboratory setting. Notwithstanding these criticisms, the participants in the professional group complimented the verisimilitude of the simulated label-checking task.

Based on the current data, two different approaches to improving performance suggest themselves: selecting label-checking personnel based on their cognitive profile or changing label-checking behaviour in such a way that the chances of individual differences having an impact on performance are reduced significantly. Methods of achieving the latter have been considered in related work (Smith-Spark et al., 2015, 2016), in which label-checking can be computer-guided to constrain the approach taken to the task such that it follows a systematic, one field at a time pattern. In this way, the cognitive load associated with label-checking is reduced and all fields of the label are checked. Methods of personnel selection for the task of labelchecking have been considered by Katz et al. (2015), discussing the use of cognitive tests to screen for prospective candidates for label-checking roles or to identify the areas of cognition in which they need to be supported in order to perform the label-checking task optimally.

\section{GENERAL DISCUSSION}

The research reported in this paper investigated human and situational factors that might influence the performance of quality control staff in detecting errors. Firstly, observational work on the packhouse floor and interviews with key quality control staff highlighted a number of factors which might result in human error, such as fatigue, cognitive load, and the ability to work efficiently in the face of distractors and interruptions. Identifying these situational factors may prove useful to industry, particularly when considering job redesign. Secondly, individual differences in information processing speed and inhibition abilities were found to contribute to differences in label-checking accuracy under laboratory conditions. At this point, it should again 
be noted that the measures tested accounted for only $19 \%$ of variance in label error detection accuracy. However, the costly nature of undetected errors (and the current gap in industry and academic knowledge in how to reduce them) should argue for the contribution of the current research to understanding human error in the quality control checking of product labels. While more research is needed to identify further contributors to label-checking performance, the understanding gained from the work reported in this paper should feed into attempts to reduce the financial and environmental costs associated with such errors. More generally, the findings should have relevance to other areas of manufacture or surveillance where humans are responsible for monitoring processes in order to detect anomalies or errors. 


\section{References}

Åkerstedt, T., \& Gillberg, M. (1990). Subjective and objective sleepiness in the active individual. International Journal of Neuroscience, 52, 29-37.

Byrne, M. D., \& Bovair, S. (1997). A working memory model of a common procedural error. Cognitive Science, 21, 31-61.

Chein, J. M., Moore, A. B., \& Conway, A. R. A. (2011). Domain-general mechanisms of complex working memory span. NeuroImage, 54, 550-559.

Cowan, N. (2008). What are the differences between long-term, short-term, and working memory? Progress in Brain Research, 169, 323-338.

Diamond, A. (2013). Executive functions. Annual Review of Psychology, 64, 135-168.

Dodhia, R. M., \& Dismukes, R. K. (2009). Interruptions create prospective memory tasks. Applied Cognitive Psychology, 23, 73-89.

Drury, C. G. (1993). Exploring search strategies in aircraft inspection. In D. Brogan, A. Gale \& K. Carr (Eds.), Visual Search 2 (pp. 101-112). London: Taylor \& Francis.

Eckstein, M. P. (2011). Visual search: A retrospective. Journal of Vision, 11, 1-36.

Friedman, N. P., \& Miyake, A. (2017). Unity and diversity of executive functions: Individual differences as a window on cognitive structure. Cortex, 86, 186-204.

Gallwey, T. J. (1982). Selection tests for visual inspection on a multiple fault-type task. Ergonomics, 25, 1077-1092.

Gallwey, T. J., \& Drury, C. G. (1986). Task complexity in visual inspection. Human Factors, $28,595-606$.

Ghylin, K. M., Drury, C. G., \& Schwaninger, A. (2006). Two-component model of security 
inspection: Application and findings. Proceedings of the 16th World Congress of Ergonomics. Maastricht, The Netherlands: International Ergonomics Association.

Jameeson, G. H. (1966). Inspection in the telecommunications industry: A field study of age arid other performance variables. Ergonomics, 9, 297-303.

Jersild, A. T. (1927). Mental set and shift. Archives of Psychology, Whole No. 89.

Katz, H. B., Smith-Spark, J. H., Wilcockson, T. D. W., \& Marchant, A. (2015). Cognitive predictors of accuracy in quality control checking. In G. Airenti, B. G. Bara, \& G. Sandini (Eds.), Proceedings of the EuroAsianPacific Joint Conference on Cognitive Science. CEUR Workshop Proceedings, 1419, 2015.

Kleiner, B. M., \& Drury, C. G. (1993). Design and evaluation of an inspection programme. Applied Ergonomics, 24, 75-82.

Lee, F. C. H., Man, S. S., \& Chan, A. H. S. (2019). Effects of magnification modes and location cues on visual inspection performance. PLOS ONE, 14(3), e0213805.

Liu, N., \& Yu, R. (2018). Social facilitation effect in search and decision-making components of visual inspection. Human Factors and Ergonomics in Manufacturing \& Service Industries. doi: https://doi.org/10.1002/hfm.20778

Luria, A. R. (1966). Higher cortical functions. New York, NY: Basic Books.

Mäntylä, T. (1994). Remembering to remember: Adult age differences in prospective memory. Journal of Gerontology, 49, 276-282.

Melchore, J. A. (2011). Sound practices for consistent visual inspection. AAPS PharmSciTech, $12,215-221$.

Melloy, B. J., Harris, J. M., \& Gramopadhye, A. K. (2000). Predicting the accuracy of visual 
search performance in the structural inspection of aircraft. International Journal of Industrial Ergonomics, 26, 277-283.

Miyake, A., \& Friedman, N. P. (2012). The nature and organization of individual differences in executive functions: Four general conclusions. Current Directions in Psychological Science, 21, 8-14.

Miyake, A., Friedman, N. P., Emerson, M. J., Witzki A. H., Howerter A., \& Wager, T. D. (2000). The unity and diversity of executive functions, and their contributions to complex "frontal lobe" tasks: A latent variable analysis. Cognitive Psychology, 41, 49-100.

Monsell, S. (2003). Task switching. Trends in Cognitive Sciences, 7, 134-140.

Rao, P., Bowling, S. R., Khasawneh, M. T., Gramopadhye, A. K., \& Melloy, B. J. (2006). Impact of training standard complexity on inspection performance. Human Factors and Ergonomics in Manufacturing, 16, 109-132.

Roth, H. L, Lora, A. N., \& Heilman, K. M. (2002). Effects of monocular viewing and eye dominance on spatial attention. Brain, 125, 2023-35.

Smith-Spark, J. H., Henry, L. A., Messer, D. J., Edvardsdottir, E., \& Zięcik, A. P. (2016). Executive functions in adults with developmental dyslexia. Research in Developmental Disabilities, 53-54, 323-341.

Smith-Spark, J. H., Katz, H. B., Marchant, A., \& Wilcockson, T. D. W. Label-checking strategies to adapt behaviour to design. ECCE '15: Proceedings of European Conference on Cognitive Ergonomics 2015. Article No. 13. ACM, New York, USA, 2015.

Smith-Spark, J. H., Katz, H. B., Marchant, A., \& Wilcockson, T. D. W. (2016). Reducing quality 
control errors by guiding behavior. Book of Proceedings of the 6th International Ergonomics Conference Ergonomics 2016 - Focus on synergy (pp. 315-322). Croatian Ergonomics Society, Zagreb, Croatia.

Smith-Spark, J. H., Katz, H. B., Marchant, A., \& Wilcockson, T. D. W. (2018). Optimal approaches to the quality control checking of product labels. International Journal of Industrial Ergonomics, 68, 118-124.

Smith-Spark, J. H, Moss, A. C., \& Dyer, K. (2016). Do baseline executive functions mediate prospective memory performance under a moderate dose of alcohol? Frontiers in Psychology, 7, Article 1325.

Snodgrass, J. G., \& Vanderwart, M. (1980). A standardized set of 260 pictures: norms for name agreement, image agreement, familiarity, and visual complexity. Journal of Experimental Psychology: Human Learning and Memory, 6, 174-215.

Taylor, W., Melloy, B., Dharwada, P., Gramopadhye, A., \& Toler, J. (2004). The effects of static multiple sources of noise on the visual search component of human inspection. International Journal of Industrial Ergonomics, 34, 195-207.

Trafton, J. G., \& Monk, C. A. (2007). Task interruptions. Reviews of Human Factors and Ergonomics, 3, 111-126.

Unsworth, N., Heitz, R. P., Schrock, J. C., \& Engle, R. W. (2005). An automated version of the operation span task. Behavior Research Methods, 37, 498-505.

Wang, M.-J. J., \& Drury, C. G. (1989). A method of evaluating inspector's performance differences and job requirements. Applied Ergonomics, 20, 181-190.

Wang, M.-J. J., Lin, S.-C., \& Drury, C. G. (1997). Training for strategy in visual search. International Journal of Industrial Ergonomics, 20, 101-108. 
Wechsler, D. (1998). The Wechsler Adult Intelligence Scale- Third Edition (UK Edition). London, UK: The Psychological Corporation.

Wilson, M. D., Farrell, S., Visser, A. W., \& Loft, S. (2018). Remembering to execute deferred tasks in simulated air traffic control: The impact of interruptions. Journal of Experimental Psychology: Applied, 24, 360-379.

Winograd, E. (1988). Some observations on prospective remembering. In M. M. Gruneberg, P. E. Morris, \& R. N. Sykes (Eds.), Practical aspects of memory: Current research and issues (Vol. 1, pp. 348-353). Chichester, UK: Wiley.

Yerkes, R. M., \& Dodson, J. D. (1908). The relation of strength of stimulus to rapidity of habitformation. Journal of Comparative Neurology and Psychology, 18, 459-482. 
Table 1

The factors identified by label-checkers as contributing to label-checking error, showing the percentage of interviewees identifying the factor as a contributor to error.

Smaller point size of the print on the label or the product specification sheet

A higher number of items on the label to be remembered to be checked

High noise levels in the packhouse

Use of black and white print for fields on the product specification sheet

The cold temperature of the packhouse 
Factors affecting label-checking accuracy

Table 2

Group means for the measures entered into the regression models (with standard deviations shown in parentheses).

\begin{tabular}{lcc}
\hline Cognitive measure & Experienced group & Novice group \\
\hline KSS Time 1 score & 3.88 & 3.80 \\
& $(1.36)$ & 135 \\
Digit-Symbol completion & 130 & $(22.35)$ \\
time (s) & $(18.44)$ & 91 \\
Go/No accuracy (\%) & 93 & $(8.60)$ \\
& $(8.06)$ & 37 \\
Operation Span absolute & 23 & $(16.54)$ \\
span score * & $(15.70)$ & 22 \\
Plus-Minus switch cost (s) & 4 & $(27.64)$ \\
& $(8.22)$ & \\
\hline KEY: * Due to equipment failure, the data for one novice participant were not recorded.
\end{tabular}


Factors affecting label-checking accuracy

Table 3

Predictors of overall label-checking accuracy.

\begin{tabular}{lcc}
\hline Cognitive measure & Partial correlation & Test statistics \\
\hline Go/No Go accuracy & .386 & $t=2.25, p=.032$ \\
Digit-Symbol completion time & -.015 & $t<1, p=.938$ \\
Operation Span absolute span score & .076 & $t<1, p=.692$ \\
Plus-Minus switch cost & -.124 & $t<1, p=.515$ \\
KSS Time 1 score & -.005 & $t<1, p=.979$ \\
\hline
\end{tabular}


Table 4

Predictors of label error detection accuracy.

\begin{tabular}{lcc}
\hline Cognitive measure & Partial correlation & Test statistics \\
\hline Go/No Go accuracy & -.236 & $t=1.28, p=.210$ \\
Digit-Symbol completion time & .464 & $t=2.82, p=.009$ \\
Operation Span absolute span score & -.110 & $t<1, p=.562$ \\
Plus-Minus shift cost & .099 & $t<1, p=.603$ \\
KSS Time 1 score & -.198 & $t=1.07, p=.295$ \\
\hline
\end{tabular}


Figure legends

Figure 1

A simplified product specification sheet. Key: $B B=$ Best before date.

Figure 2

A fresh produce label displaying seven bits of information (namely, the product name, best before date, grower name, country of origin, quantity or weight, barcode, and a flash label).

Figure 3

A flowchart of the label-checking process, giving details of the task, task performer and person in charge.

Figure 4

Participant group x block of trials interaction for percent detected label errors. 